\title{
Diagnostic Efficacy of Magnetization Transfer Contrast in Comparison with Fluid Attenuated Inversion Recovery Sequences for Evaluating Brain Tuberculoma in South Indians
}

\author{
Lavanya $^{1}$, Abubacker Sulaiman Farook ${ }^{2}$, Raj Kumar ${ }^{3}$, Ashraf Ahmed B ${ }^{4}$, Einstein Raju ${ }^{5}$, Farooque MC \\ ${ }^{1}$ Final year PG, ${ }^{2}$ Professor, ${ }^{3}$ Senior Resident, ${ }^{4}$ Associate Professor, ${ }^{5}$ Associate Professor, ${ }^{6}$ Second Year PG, Department of \\ Radio-diagnosis, Chettinad Hospital and Research Institute, Kelambakkam, Chennai, TN, India 603 103, India
}

Corresponding author: Prof. Dr. Abubacker Sulaiman F, Professor Department of Radiology and Imaging Sciences, Chettinad Hospital and Research Institute, India

DOI: 10.21276/ijcmsr.2018.3.2.8

How to cite this article: Lavanya, Abubacker Sulaiman Farook, Raj Kumar, Ashraf Ahmed B, Einstein Raju, Farooque MC. Diagnostic efficacy of magnetization transfer contrast in comparison with fluid attenuated inversion recovery sequences for evaluating brain tuberculoma in South Indians. International Journal of Contemporary Medicine Surgery and Radiology. 2018;3(2):B31-B35.

\section{A B S T R A C T}

Introduction: Brain tuberculosis results from Bacterial spread which primarily exists in the body. Tuberculomas are firm, avascular, spherical masses, with size varying between $2 \mathrm{~cm}$ and $10 \mathrm{~cm}$ in diameter. Aim of the study was to evaluate and contrast T1-weighted magnetization transfer (MT) with Fluid attenuated inversion restoration (FLAIR) picture for analyzing conspicuity and number of patches in individuals with brain tuberculoma in south Indians.

Material and Methods: In all 17 cases with brain tuberculoma subjected to MR evaluation using quick spin-echo (FSE) T2, spin-echo (SE) T1, T1-weighted MT and FLAIR Imaging. Post-contrast T1-weighted MT images was taken as the defacto conventional for evaluating the variety of lesions.

Results: Tuberculoma found both on T1-weighted MT and FLAIR images that were examined for the boundaries to be described, and were separated into two categories according to presence (group 1) or absence (group 2) of perilesional oedema noticeable on FLAIR images. The mean indication strength of the boundaries of the patches and nearby oedema or brain parenchyma was examined qualitatively and quantitatively.

Conclusion: FLAIR images is not helpful in the study of Brain tuberculomas in contrast to T1-weighted MT picture, as it neither leads to the depiction of lesion nor analyzes the true disease burden.

Keywords: Brain Tuberculoma, Fluid Attenuated Inversion Recovery, Magnetization Transfer Contrast

\section{INTRODUCTION}

Tuberculosis (TB) is a multi-systemic infectious disease caused by different species of mycobacteria, usually Mycobacterium tuberculosis in humans. Tuberculosis of the nervous system can attack the meninges, brain, spinal cord, cranial and peripheral nerves, ears, and eyes. Brain tuberculosis results from spread of the bacteria which primarily exists in the body. Tuberculomas are firm, avascular, spherical masses, with size varying between $2 \mathrm{~cm}$ and $10 \mathrm{~cm}$ in diameter. They are well circumscribed, and the compressed surrounding brain tissue shows edema and gliosis. The inside of the masses contains necrotic areas of caseation in which tubercle bacilli may be found. Tuberculous brain abscess is a distinct condition. ${ }^{1-3}$

Fluid-attenuated inversion recovery (FLAIR) is a special inversion recovery pulse sequence with a long repetition time (TR) and echo time (TE), and an inversion time (TI) that effectively nulls signals from the cerebrospinal fluid (CSF). 4,5 Intravenous magnetic resonance (MR) contrast agents are frequently used to improve lesion detection and characterization of central nervous system (CNS) disorders. The commonly used contrast agent, Gd, shortens both the $\mathrm{T} 1$ and $\mathrm{T} 2$ relaxation times of tissues in which it has accumulated. ${ }^{6-8}$ Use of FLAIR images in other infections and inflammation related condition has given appealing results; however, a few researches has revealed drawbacks in circumstances such as brain infarction and MS. Combining all the specific sequences in MR for the evaluation of neurological diseases is not possible because of time limitation factors and the patients with such conditions are very sick to be held up against extended evaluation. ${ }^{9-11}$ This research was meant to evaluate FLAIR and T1-weighted MT image for identifying the conspicuity of the walls of a lesion in the existence and lack of the perilesional oedema and also to quantify the infection load in cases of brain tuberculoma.

\section{MATERIAL AND METHODS}

This potential study done in Department of Radio-diagnosis, Chettinad Hospital and Research Institute, Kelambakkam, Chennai, included 17 patients, 9 males and 8 females, with age groups ranging from 6 to 38 years with variegated clinical 
demonstration of symptoms including high temperature, meningeal discomfort, and focal neurological deficits corresponding to the site of lesion rigid neck or convulsions. Informed consent was taken from all oriented individuals over 18 years of age. In the case of children and affected individuals with altered mental status, approval was taken from the next of kin. In 5 cases, tuberculomas were associated with tuberculous meningitis (TBM). The biochemical parameters of CSF were also collected.

In 2 cases, several tubercles were seen on the ependymal lining during endoscopic third ventriculostomy as part of control, and biopsy verified the analysis. In 4 cases, the appropriate tuberculoma was verified on histopathology. In the rest of cases, the analysis was depending on the appearance of miliary pulmonary $\mathrm{TB}$ on chest skiagram $(n=3)$, quantification of magnetization exchange rate (MTR) from the walls $(n=3)$ and reaction to particular treatment on follow up all these patients.

MRI was conducted with a GE Signa HDx 1.5 tesla MR device (Signa replicate rate plus, Common Power Healthcare Techniques, The usa, MN, USA) built with an definitely guarded whole-body attractive area slope set enabling up to $33 \mathrm{mT} / \mathrm{m}$ and using quadrature go coils. The schedule image analysis involved quick spin-echo (FSE) T2-weighted (repetition time (TR)/echo time (TE)/number of excitations (n)Z4900 ms/85 ms/3) and spinecho (SE) T1-weighted (TR/TE/flip angle/nZ $1300 \mathrm{~ms} / 14 \mathrm{~ms} / 658 / 2$ ) and liquid attenuated inversion restoration (TR/TE/nZ9000 ms/120 $\mathrm{ms} / 1$; inversion time, $2200 \mathrm{~ms}$ ) series. All images were obtained in axial planes with $5-\mathrm{mm}$ thickness, a $0.5-\mathrm{mm}$ interslice gap and a $256^{*} 256$ matrix. The pulse sequence was used for MT comparison containing an off-resonance vividness beat be fore the 908 excitation beat to absorb the magnetization of protons with limited movement. Fermi shaped RF impulses of $16 \mathrm{~ms}$ length, with a turn position of 6708 and an off-resonance regularity of $1200 \mathrm{~Hz}$, were used to acquire the MT impact. For T1-weighted MT images, only vividness beat was added; the other factors were similar to the traditional SE T1-weighted pictures. Postcontrast T1-weighted MT picture and routine T1C was also conducted one after the other for all patients,. Contrast agent- Meglumine Gadoterate (DOTAREM) $0.5 \mathrm{mmol} / \mathrm{ml}$ is injected intravenously, for better visualization of the lesion on the background of normal parenchyma.

All identified lesions were visualized on FLAIR, post contrast T1 and post-contrast T1-weighted MT picture. The latter was regarded the defacto conventional for this analysis. Lesions noticeable on FLAIR as well as on post contrast T1-weighted MT picture were split into two categories on the factors for existence or lack of perilesional oedema noticeable on FLAIR imaging; group 1 composed those with and group 2 those without perilesional oedema. Qualitative and quantitative assessments were conducted individually by two radiologists. In equivocal situations, the help of a knowledgeable Radiologist was desired. Distinction of the walls of primary lesion from oedema were documented in every situation, on both MT and FLAIR sequences, with initial appearance and by quantitative research. The signal intensity strength was evaluated with a user-friendly visual customer interface that originated in-house using Visual Studio room (version 6.0). The overall mean signal intensity was derived by putting three to five areas of interest (ROIs) of the walls of the lesion, nearby brain parenchyma or oedema, respectively, on both FLAIR and T1-weighted MT images. Outcomes displaying the best-defined walls on one series were co-registered with corresponding results on the other series, using the computerized picture signing up (AIR) system.

\section{STATISTICAL ANALYSIS}

Probability ( $p$ ) values were computed for mean signal intensities from wall and surrounded parenchyma with and without edema in both groups, to look for any significant differences; $p$ values of less than 0.05 were regarded as statistically significant. Interobserver variation was calculated with weighted $\mathrm{k}$ statistics were used for statistical Analysis.

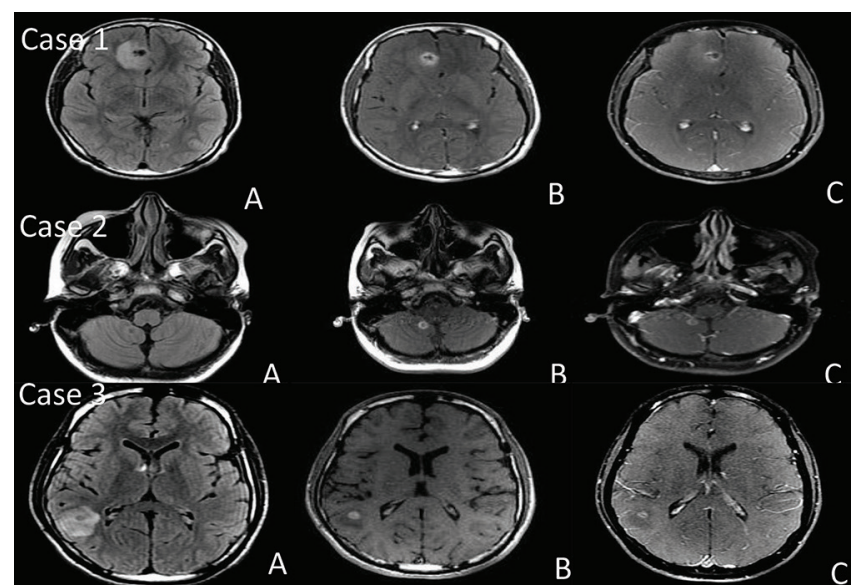

Case-1:(A) T2 FLAIR MRI image showing a well defined rounded hypointense lesion with mild perilesional edema noted in sub-cortical aspect of right frontal lobe. (B) Post contrast MT image showing intense ring enhancement of the corresponding lesion. (C) Contrast Enhanced T1WI showing rim enhancement of the lesion. Compared to all 3 sequences, contrast enhanced MT Image showed better delineation of the lesion. The ring enhancement produced on MT was more intense than routine T1C. Case-2: (A) T2 FLAIR MRI image showing a well defined hypointense lesion with no significant edema noted in the right cerebellar tonsil. (B) post contrast MT image shows a corresponding lesion showing smooth, regular, intense ring enhancement. (C) Post Gadolinium enhanced T1WI showing ring enhancing lesion. Additionally, the patient aslo had leptomeningeal and tentorial enhancement (TB Meningitis) which was missed on MT, But was recognized on T1C. Case-3: A 30 year old male patient presented with headache and few episodes of convulsions. MRI brain with contrast taken, which revealed, (A) T2 FLAIR MRI image showing a well defined spherical hypointense lesion with surrounding peri-lesional edema noted involving the juxta-cortical white matter of right parietal lobe. (B) post contrast MT image shows corresponding lesion as showing smooth, regular rim enhancement. (C) Post contrast enhanced T1WI Showing smooth, complete ring enhancing lesion. Compared to MT image, the lesion appears less intense and subtle on T1C. 


\section{RESULTS}

None of the patients included in the study was positive for HIV. The CSF results in instances of tuberculous meningitis were as follows: mean mobile solidity (WBC) 190 cells $/ \mathrm{mm} 3$ (range 169 to 211 cells/mm3), mean sugar $34 \mathrm{mg} \%$ (range $28.5 \%$ to $37 \%$ ) and mean proteins $196 \mathrm{mg} \%$ (range $179 \%$ to $208 \mathrm{mg} \%$ ). The ELISA was beneficial for IgM and $\mathrm{IgG}$ in all tuberculous meningitis situations.

\section{Case-1}

A 23 year old male patient presented with seizure, preceded by headache and fever. MRI Brain was done, the following sequences revealed

(A) T2 FLAIR MRI image showing a well defined rounded hypointense lesion with mild perilesional edema noted in sub-cortical aspect of right frontal lobe.(B) Post contrast MT image showing intense ring enhancement of the corresponding lesion. (C)Contrast Enhanced T1WI showing rim enhancement of the lesion. Compared to all 3 sequences, contrast enhanced MT Image showed better delineation of the lesion. The ring enhancement produced on MT was more intense than routine $\mathrm{T} 1 \mathrm{C}$.

\section{Case-2}

A 17 Year old female patient presented with headache, neck stiffness and gait abnormalities. she also had past history of Tuberculosis and claims to have completed the course of treatment. MRI Brain was done.

(A) T2 FLAIR MRI image showing a well defined hypointense lesion with no significant edema noted in the right cerebellar tonsil.(B) post contrast MT image shows a corresponding lesion showing smooth, regular, intense ring enhancement. (C) Post Gadolinium enhanced T1WI showing ring enhancing lesion. Additionally, the patient aslo had leptomeningeal and tentorial enhancement (TB Meningitis) which was missed on MT, But was recognized on $\mathrm{T} 1 \mathrm{C}$.

\section{Case-3}

A 30 year old male patient presented with headache and few episodes of convulsions. MRI brain with contrast taken, which revealed,

(A) T2 FLAIR MRI image showing a well defined spherical hypointense lesion with surrounding peri-lesional edema noted involving the juxta-cortical white matter of right parietal lobe. (B) post contrast MT image shows corresponding lesion as showing smooth, regular rim enhancement. (C) Post contrast enhanced T1WI Showing smooth, complete ring enhancing lesion. Compared to MT image, the lesion appears less intense and subtle on T1C.

\section{Lesion detection}

All lesions seen on FLAIR were also noticeable on T1weighted MT imaging: 49 lesions were recognized in 17 subjects on post-contrast T1-weighted MT picture, of which all 49 were recognized on contrast enhanced T1-weighted images (100\%) and 27 on FLAIR pictures (66.7\%). The MT rate from the walls of these lesions varied from $19 \%$ to $23 \%$. The overall submission of the 49 lesions recognized on postcontrast T1-weighted MT pictures was: frontal 7, parietal 8 temporal 7, occipital 5, thalami basal ganglia 9 cerebellum
5 and brain stem 8 There was conflict between the two radiologists regarding the variety of lesions on FLAIR in one patient, which was settled with the help of the knowledgeable neuroradiologist.

On Comparing pre and post-contrast T1-weighted MT images, 13 more lesions were noticeable than on pre-contrast T1-weighted MT picture. The submission of the skipped lesions was frontal 4, parietal 3, occipital 2, temporal 3, thalami 1, cerebellum 2 and brain stem 2; 1 of these were situated in the cortex in the Grey-white interface 2 in basal ganglia. All the unidentified lesions were less than $5 \mathrm{~mm}$ across.

Of the 27 lesions seen on FLAIR and on T1-weighted MT picture, 19 had peri-lesional edema and constituted team 1 . The remaining 8 lesions did not display any associated edema and constituted team 2; 11 lesions were not discreetly imaged or faintly seen on FLAIR picture although they were very well seen on T1-weighted MT picture (Fig. 2). The submission of these lesions was frontal 8, parietal 7, temporal 4 , basal ganglia 2 and cerebellum 6 . None of these lesions were associated with peri-lesional edema and all were less than $5 \mathrm{~mm}$ across.

\section{Lesion conspicuity}

For distinction of the walls from the adjacent normal parenchyma with or without peri-lesional edema, T1weighted MT picture was greater than T1C Image for qualitative and quantitative research. In group 1 (Figs. 1 and 3 ), the distinction in signal intensity between the walls of the lesion and the peri-lesional edema was Very well delineated on MT Images when compared to T1C images. However, all the imaged seen on Post-contrat MT were picked up on $\mathrm{T} 1 \mathrm{C}$, with some lesions showing faint enhancement than on MT.

In group 2 (Figs. 2), the signal intensity between the walls of the lesion and the adjacent parenchyma was established on both T1-weighted MT and FLAIR images. But however, the MT Images were more intense with better visualization of the walls when compared to FLAIR.

\section{Inter Observer Variability}

The $\mathrm{k}$ score for inter observer reproducibility of the classification of lesion conspicuity was 0.59 for the FLAIR and 0.80 for T1-weighted MT images.

\section{DISCUSSION}

Magnetization Transfer Imaging is a technique for improving image contrast in Magnetic Resonance (MR) imaging. It is based on application of off-resonance radio-frequency pulses and observing their effects on MR images. ${ }^{11}$ The clinical manifestations of tuberculomas depend on their locationand this may be supratentorial, in the brainstem, elsewhere in the posterior fossa, or even in the sella. Contrast-enhanced FLAIR imaging has many advantages for intracranial disease manifestations. ${ }^{12}$ Hence; we planned study to evaluate FLAIR and T1-weighted MT image for identifying the conspicuity of the walls of a lesion in the existence and lack of the perilesional oedema and also to quantify the infection load in cases of brain tuberculoma.

In our study, plain T1-weighted MT picture revealed relatively 
less lesions in comparison with post-contrast T1-weighted MT and routine T1C. Lesions that went unnoticed on T1weighted MT picture were less than $5 \mathrm{~mm}$ in diameter; 6 out of the 13 skipped lesions were situated either in the cortex or deep grey nuclei. MT ratios of tuberculoma and the cortex or the brain stem where the lesion was situated, probably did not produce enough distinction in the signal strength for the lesion to become noticeable. The little dimensions of these lesions also provided towards their nonvisualization on T1-weighted MT picture. Furthermore, it is evident from histopathological studies that FLAIR imaging may fail to show oedema in the tissues surrounding it. This lack of hyperintensity on FLAIR imaging could possibly be attributed to the presence of macromolecules in the oedema that may escape into the parenchyma following the breach of the blood-brain barrier, and affect the tissue relativity. In addition, FLAIR imaging has other drawbacks that have been reported in literature, such as hyperintense artefacts related to CSF flow, and inadequate depiction of lesions in the basal ganglia and brain stem. T1-weighted MT imaging displays a hyper-intense rim in tuberculomas, thus aiding their characterization. This wall is composed of cellular infiltrate, granulomas, Langhans' giant cells and gliosis or fibrosis, and is inconsistently seen in other lesions of similar appearance, such as neuro cysticercosis and tumors. The depiction of this wall was poor in FLAIR imaging in the presence of oedema in our series. A similar lack of demonstration of the wall was also noted on conventional T2-weighted images. This occurs because the hyper intensity of the wall and the oedema merges together in FLAIR imaging. In the absence of perilesional oedema, FLAIR imaging showed the wall, but delineation was much better on T1-weighted MT imaging, resulting in better characterization of the lesion. ${ }^{13,14}$

Saxena S et al compared T1-weighted magnetization transfer (MT) with fluid attenuated inversion recovery (FLAIR) imaging for evaluating conspicuity and number of lesions in individuals with brain tuberculoma. Tuberculomas detected both on T1-weighted MT and FLAIR imaging were examined for the wall to be defined, and were divided into two groups on the basis of presence (group 1) or absence (group 2) of perilesional oedema visible on FLAIR imaging. The mean signal intensity of the wall of the lesions and adjacent oedema or brain parenchyma was analyzed qualitatively and quantitatively. The number of lesions detected on T1weighted MT was higher than on FLAIR imaging (209 versus 163). Conspicuity in both groups was better on T1weighted MT images qualitatively as well as quantitatively. The difference in the signal intensity of the wall of the lesion and perilesional oedema was statistically significant only on T1-weighted MT images in group 1 ( $p=0.0003$ versus 0.3 ), whereas in group 2 it was statistically significant both on T1weighted MT and FLAIR imaging ( $\mathrm{p}=0.009$ versus 0.05 ). FLAIR imaging is not helpful in the examination of brain tuberculomas compared with T1-weighted MT imaging, as it neither contributes to the characterization of lesion nor assesses the true disease load. ${ }^{15}$ Kubota $\mathrm{T}$ et al investigated the relationship between the degree of contrast enhancement in fluid-attenuated inversion recovery (FLAIR) sequences and tumor signal intensity on T2-weighted images and concluded that the signal intensity in FLAIR sequences is largely influenced by both $\mathrm{T} 1$ and $\mathrm{T} 2$ relaxation time; there is a close relationship between the signal intensity of brain tumors on T2-weighted images and the degree of contrast enhancement on FLAIR sequences. ${ }^{16}$

\section{CONCLUSION}

We determine that the FLAIR images is not certainly useful in the evaluation of brain tuberculoma in comparison to post contrast T1-weighted MT imaging; It is neither superior in the depiction of tuberculous lesions, nor for illustrating the real disease condition. T1C however equally picked up lesions as in MT, but the lesion is more intense on Post contrast MT. It is neither superior in the depiction of tuberculous lesions, nor for illustrating the real disease condition.

\section{REFERENCES}

1. De Coene B, Hajnal JV, Gatehouse P, Longmore DB, White SJ, Oatridge A, et al. MR of the brain using fluidattenuated inversion recovery (FLAIR) pulse sequences. AJNR Am J Neuroradiol. 1992;13(1):1555-1564.

2. Kremer S, Abu Eid M, Bierry G, Bogorin A, Koob M, Dietemann JL, et al. Accuracy of delayed postcontrast FLAIR MR imaging for the diagnosis of leptomeningeal infectious or tumoral diseases. J Neuroradiol. 2006;33(2):285-291.

3. Melhem ER, Bert RJ, Walker RE. Usefulness of optimized gadolinium-enhanced fast fluid-attenuated inversion recovery MR imaging in revealing lesions of the brain. AJR Am J Roentgenol. 1998;171(4):803-807.

4. Bozzao A, Floris R, Fasoli F, Fantozzi LM, Colonnese C, Simonetti G. Cerebrospinal fluid changes after intravenous injection of gadolinium chelate: assessment by FLAIR MR imaging. Eur Radiol. 2003;13(6):592597.

5. Hajnal JV, Bryant DJ, Kasuboski L, Pattany PM, De Coene B, Lewis PD, et al. Use of fluid attenuated inversion recovery (FLAIR) pulse sequences in MRI of the brain. J Comput Assist Tomogr. 1992;16(3):841844.

6. Smirniotopoulos JG, Murphy FM, Rushing EJ, Rees JH, Schroeder JW. Patterns of contrast enhancement in the brain and meninges. Radiographics. 2007;27(5):525551.

7. Kim EY, Kim SS, Na DG, Roh HG, Ryoo JW, Kim HK. Sulcal hyperintensity on fluid-attenuated inversion recovery imaging in acute ischemic stroke patients treated with intra-arterial thrombolysis: iodinated contrast media as its possible cause and the association with hemorrhagic transformation. J Comput Assist Tomogr. 2005;29(1):264-269.

8. Smirniotopoulos JG, Murphy FM, Rushing EJ, Rees JH, Schroeder JW. Patterns of contrast enhancement in the brain and meninges. Radiographics. 2007;27(5):525551.

9. Fukuoka H, Hirai T, Okuda T, Shigematsu Y, Sasao A, Kimura E, et al. Comparison of the added value of contrast-enhanced 3D fluid-attenuated inversion recovery and magnetization-prepared rapid acquisition of gradient echo sequences in relation to conventional postcontrast T1-weighted images for the evaluation of 
leptomeningeal diseases at 3T. AJNR Am J Neuroradiol. 2010;31(4):868-873.

10. Mathews VP, Caldemeyer KS, Ulmer JL, Nguyen H, Yuh WT. Effects of contrast dose, delayed imaging, and magnetization transfer saturation on gadoliniumenhanced MR imaging of brain lesions. J Magn Reson Imaging. 1997;7(2):14-22.

11. Mathews VP, Caldemeyer KS, Lowe MJ, Greenspan SL, Weber DM, Ulmer JL. Brain: gadolinium-enhanced fast fluid-attenuated inversion-recovery MR imaging. Radiology. 1999;211(5):257-263.

12. Jeon JY, Choi JW, Roh HG, Moon WJ. Effect of imaging time in the magnetic resonance detection of intracerebral metastases using single dose gadobutrol. Korean J Radiol. 2014;15(7):145-150.

13. Rydberg JN, Hammond CA, Grimm RC, Erickson BJ, Jack CR, Jr, Huston J, 3rd, et al. Initial clinical experience in MR imaging of the brain with a fast fluid-attenuated inversion-recovery pulse sequence. Radiology. 1994;193(2):173-180.

14. Sage MR, Wilson AJ, Scroop R. Contrast media and the brain. The basis of $\mathrm{CT}$ and MR imaging enhancement. Neuroimaging Clin N Am. 1998;8(4):695-707.

15. Saxena S1,Prakash M,Kumar S, Gupta RK.Comparative evaluation of magnetization transfer contrast and fluid attenuated inversion recovery sequences in brain tuberculoma. Clin Radiol. 2005;60(7):787-93.

16. Kubota T1, Yamada K, Kizu O, Hirota T, Ito H, Ishihara K, Nishimura T. Relationship between contrast enhancement on fluid-attenuated inversion recovery MR sequences and signal intensity on T2-weighted MR images: visual evaluation of brain tumors. J Magn Reson Imaging. 2005;21(6):694-700.

Source of Support: Nil; Conflict of Interest: None

Submitted: 28-04-2018; Published online: 01-05-2018 